\title{
Estudio del Clima Organizacional en Energías S.A. durante 2011
}

\section{Resumen}

Este articulo presenta los resultados de una investigación desarrollada por un grupo de estudiantes del curso Opción de Grado, del programa de Administración de Empresas de la Universidad Nacional Abierta y a Distancia (UNAD) en el CEAD de Zipaquirá con el fin de hacer un diagnostico del clima laboral de la empresa Energías S. A., que reflejase la percepción y los sentimientos del personal de la empresa en relación con la orientación organizacional, la imagen corporativa, los clientes externos, las condiciones de trabajo, la gestión del talento humano y las relaciones de equipo, con el fin de facilitar la labor del Área de Recursos Humanos en la toma de decisiones orientadas al mejoramiento del clima laboral.

Con el trabajo realizado se evidencio que el personal tiene un gran sentido de pertenencia para con la empresa; además, que existen condiciones de trabajo, relaciones de equipo y profesionalismo adecuados. Se deben mejorar, sin embargo, algunos aspectos de comunicación y el equilibro del personal entre la familia y el trabajo.

Palabras claves: Clima organizacional, condiciones de trabajo, relaciones de equipo, imagen corporativa, orientación organizacional.

\section{Abstract}

This article presents the results from a search developed by a group of students of Opción de Grado, from the program Administración de Empresas in CEAD Zipaquirá at Universidad Nacional Abierta y a Distancia (UNAD), with the purpose of make a diagnosis about the Work climate at Energías S.A. reflecting the employees perception and feelings related to the organizational orientation, corporative image, extern clients, work conditions, human talent management, team relations and balance between family and job in order to facilitate the Human Resources Area work when making decisions directed to improve the work climate.

With this work, it was evident that the employees have a great sense of membership with the company; there are some appropriate work conditions, team relationships and professionalism. There are things like communication and the employees balance between family and job, which should be improved.

Keywords: Work climate, Job Conditions, Team relationships, corporative image, organizational orientation.

Recibido: 16 de Abril de 2012

Aceptado: 25 de Julio de 2012

\footnotetext{
1 Administradora de Empresas. Especialista en pedagogía para el desarrollo del aprendizaje autónomo, MBA. Tutora y directora del curso Promoción de ventas. UNAD ECACEN Zipaquirá Correo electrónico mery.behrentz@unad.edu.co. Participante del grupo G’Kaira
} 


\section{Introducción}

El clima laboral está vinculado a la calidad de vida dentro de una organización, constituye una percepción, y, como tal, debe ser implementado en la forma de un análisis dentro de los aspectos más relevantes, junto con las expectativas del personal respecto a la empresa. Este clima se encuentra influenciado por las características del sistema organizacional, genera motivaciones o frustraciones entre los miembros, según su comportamiento, el cual tiene una gran variedad de consecuencias para la organización en cuanto a la productividad, la satisfacción, la rotación, la adaptación y el cumplimiento de objetivos, entre otros.

Según Goncalves (1997) "El clima laboral es un filtro o un fenómeno interviniente que media entre los factores del sistema organizacional (estructura, liderazgo, toma de decisiones), y las tendencias motivacionales que se traducen en un comportamiento que tiene consecuencias sobre la organización en cuanto a productividad, satisfacción, rotación, ausentismo, etc. Por lo tanto, evaluando el Clima Organizacional se mide la forma como es percibida la organización".

"El clima laboral es el medio ambiente humano y físico, es el conjunto de variables, cualidades, atributos o propiedades relativamente permanentes de un ambiente de trabajo concreto. Está relacionado con los comportamientos de las personas, con su manera de trabajar y relacionarse, con su interacción con la empresa, con el liderazgo del directivo, con las máquinas que se utilizan y con la propia actividad de cada uno".

A partir de estas definiciones se puede inferir que el concepto de percepción adquiere una importancia relevante, pues según Robins (1999) "el clima laboral está determinado por las percepciones que el trabajador tiene sobre los atributos de la organización, es decir cuál es la "opinión" que los trabajadores y directivos se forman de la organización a la que pertenecen".

Carlos Méndez, (2006) afirma que el clima organizacional se relaciona de manera importante con la determinación de la cultura organizacional de una empresa, si se entiende como cultura organizacional el patrón general de actitudes, conductas, creencias y valores compartidos por los miembros de una organización.

El clima laboral no es algo que se pueda ver o tocar, pero está ahí, presente. Las organizaciones son únicas; cada una tiene su propia cultura, su tradición y sus métodos de acción, todos los cuales, en su totalidad, constituyen el clima laboral. Este influye en la motivación, el funcionamiento y la satisfacción en el trabajo. Puede generar expectativas acerca de las consecuencias que tienen las acciones. Los empleados esperan ciertas recompensas, satisfacciones y frustraciones con base en la percepción en el clima laboral.
Se puede caracterizar el clima por ser favorable, desfavorable o neutral. En general todos quieren un clima favorable de trabajo, pues beneficia en muchos aspectos; entre ellos la satisfacción laboral, que conlleva a un buen desempeño y un mejor rendimiento.

Buscando comprender mejor el concepto de clima organizacional es necesario resaltar los siguientes elementos a ese respecto:

- El clima se refiere a las características del medio ambiente de trabajo.

- Dichas características son percibidas directa o indirectamente por los trabajadores que se desempeñan en ese medio ambiente.

- El clima tiene repercusiones en el comportamiento laboral.

- El clima es una variable interviniente que media entre los factores del sistema organizacional y el comportamiento individual.

Tales características de la organización son relativamente permanentes en el tiempo, se diferencian de una organización a otra y de una sección a otra dentro de una misma empresa.

El clima, junto con las estructuras y las características organizacionales, así como los individuos que la componen, forman un sistema interdependiente altamente dinámico.

Para medir la percepción del clima Likert tiene en cuenta ocho dimensiones:

1. Los métodos de mando. La forma como se utiliza el liderazgo para influir en los empleados.

2. Las características de las fuerzas motivacionales. Los procedimientos que se instrumentan para motivar a los empleados y responder a sus necesidades.

3. Las características de los procesos de comunicación. La naturaleza de los tipos de comunicación en la empresa, así como la manera de ejercerlos.

4. Las características de los procesos de influencia. La importancia de la interacción superior/subordinado para establecer los objetivos de la organización.

5. Las características de los procesos de toma de decisiones. La pertinencia de las informaciones en que se basan las decisiones así como el reparto de funciones.

6. Las características de los procesos de planificación. La forma como se establece el sistema de fijación de objetivos o directrices.

7. Las características de los procesos de control. El ejercicio y la distribución del control entre las instancias organizacionales. 
8. Los objetivos de rendimiento y de perfeccionamiento. La planificación así como la formación deseada.

\section{Métodos}

Para medir el clima organizacional en la empresa Energías S. A., se hizo un estudio de tipo descriptivo, el cual se usa a menudo en el área de las ciencias administrativas. Según Méndez (2006) la descripción es un subproducto de la observación que presenta los hechos tal como ocurren, los agrupa y los convierte en información; también utiliza métodos de encuesta y obser- vación. Además, Angura y Arnau (1995), exponen que la metodología de encuesta se basa fundamentalmente en recoger información aplicando un cuestionario para conocer los sentimientos, las motivaciones, los planes, etc., de unos individuos; por tal razón el enfoque metodológico utilizado es cualitativo.

Para realizar la investigación participaron 70 personas; todas ellas, empleadas de la empresa Energías S. A., con 3 tipos diferentes de contrato pertenecientes a los niveles directivo, asesor y técnico. (Tabla 1.)

$\begin{array}{lccllllll}\text { Libre Nombramiento y Remoción } & 8 & 11 \% & \text { Nivel Directivo } & 3 & 4 \% & \text { Nivel Asistendal } & 9 & 13 \% \\ \text { Carrera Administrativa } & 32 & 46 \% & \text { Asesor } & 3 & 4 \% & \text { N/S-N/R } & 10 & 14 \% \\ \text { Provisional } & 20 & 29 \% & \text { Nivel Profesional } & 31 & 44 \% & & \\ \text { N/S - N/R } & 10 & 14 \% & \text { Nivel Ténico } & 14 & 20 \% & \end{array}$

Tabla 1. Distribución de la muestra.

Inicialmente se hizo la revisión de la parte teórica acerca del clima organizacional. A continuación se realizaron las encuestas al personal de la empresa para identificar las características del recurso humano y del clima organizacional existente. Lo último fue validar la información de lo existente en la empresa, con base en la parte teórica.

La recolección de datos se llevo a cabo a través de un instrumento de medición que registró todos los datos y la información necesarios para el desarrollo de la investigación. El instrumento se aplicó a todas las personas que laboran en la empresa, y basado en un cuestionario de 77 preguntas según la metodología Likert, que es un conjunto de afirmaciones ante las cuales los encuestados deberán manifestar su grado de acuerdo o de desacuerdo, según la siguiente escala:

$$
\begin{aligned}
& T D=\text { total desacuerdo * Nunca } 1 \\
& D=\text { desacuerdo * Casi nunca } 2 \\
& A=\text { acuerdo * Casi siempre } \\
& T A=\text { Total acuerdo * Siempre }
\end{aligned}
$$

El cuestionario (Anexo 1) midió ocho variables respecto al clima organizacional de la empresa: orientación organizacional; imagen corporativa, clientes externos; condiciones de trabajo; gestión de talento humano; relaciones de equipo; profesionalismo y equilibrio familia- trabajo. Las preguntas del cuestionario son de tipo cerrado, ya que disponen de categorías y de alternativas de respuestas.
El cuestionario fue contestado por los participantes de forma voluntaria, se les garantizó la confidencialidad de la información en las propias instalaciones de la empresa.

\section{Resultados}

El objetivo de la sistematización y el análisis de la información fue conocer el estado del clima laboral en la empresa Energías S. A., teniendo en cuenta las variables relación con la empresa, condiciones de trabajo y relaciones de equipo.

\section{Relación con la empresa}

Es la percepción por parte de los miembros de la empresa acerca de la existencia de un ambiente de trabajo grato y de buenas relaciones sociales, tanto entre pares como entre jefes y subordinados. Este eje comprende las variables orientación organizacional, imagen corporativa y clientes externos.

Respecto a la orientación organizacional, dicha variable se indagó en las preguntas 1 - 6 y así se evidenció que el $95 \%$ de los empleados conoce la razón de su puesto de trabajo; además, el $76 \%$ de los participantes considera que la misión, la visión y los objetivos de la empresa son claros, y el $48 \%$ de los participantes considera que la empresa tiene en cuenta los intereses y las preocupaciones de los empleados para tomar decisiones importantes (Gráfica 1). 


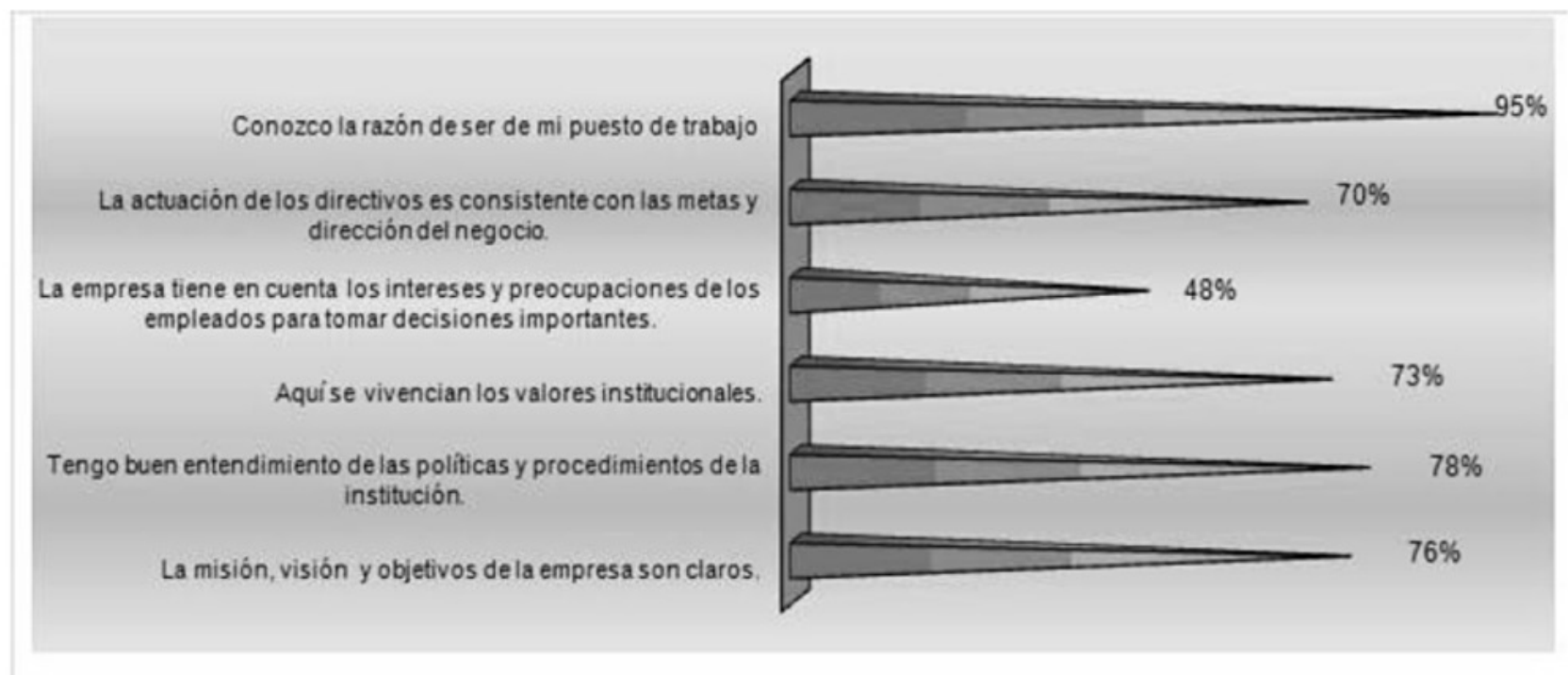

\section{Gráfica 1. Resultados en cuanto a orientación organizacional.}

Fuente: Construcción de la autora

La imagen corporativa fue revisada en las preguntas 7-14 se evidenciaron como aspectos relevantes que el $90 \%$ de los participantes se sienten orgullosos de trabajar en la empresa, el $86 \%$ considera que vale la pena dar lo mejor de si mismos para el desarrollo de la empresa y el 63\% siente que esta en un lugar estable para trabajar (Gráfica 2.)

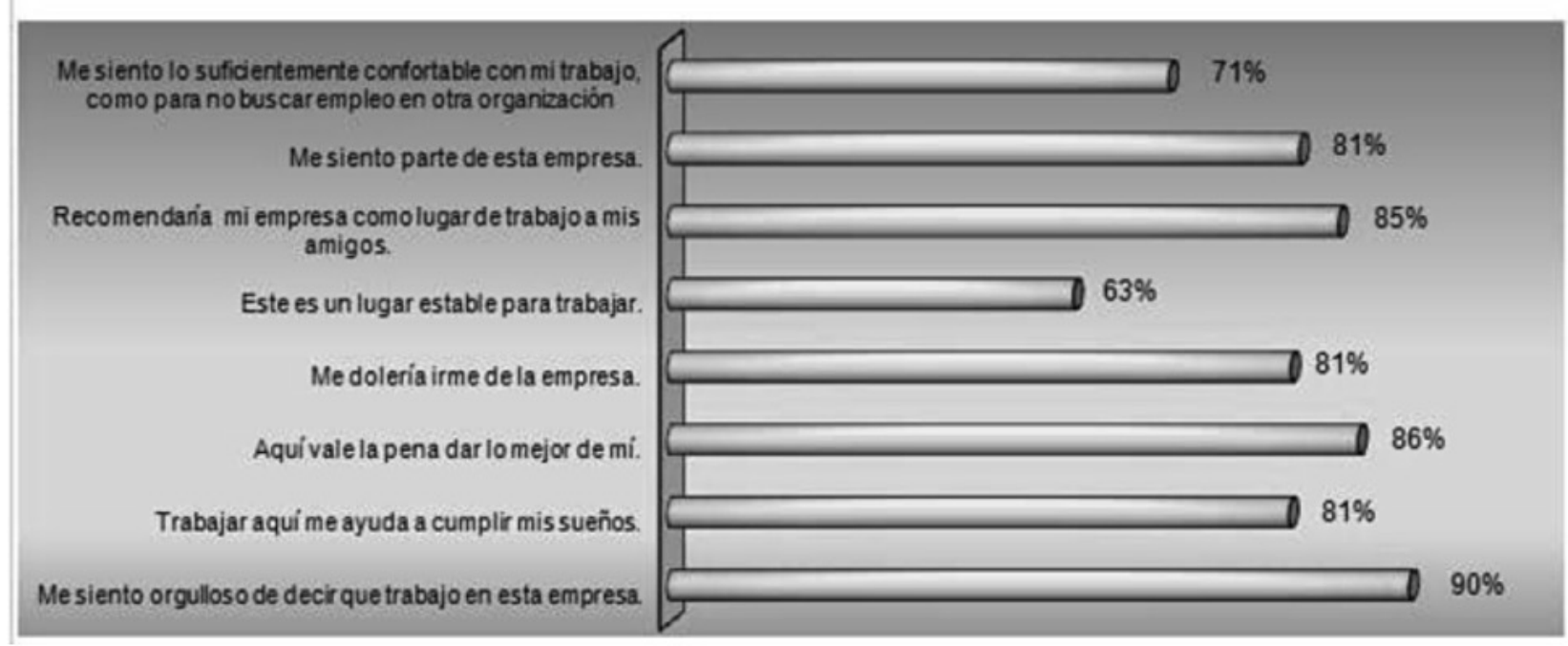

\section{Gráfica 2. Resultados en cuanto a imagen corporativa}

\section{Fuente: Construcción de la autora}

En la variable correspondiente a clientes externos, evaluada en las preguntas 15-19, se halló que el 93\% de los clientes están satisfechos con el servicio prestado, el $75 \%$ considera que la empresa innova constantemente y el $87 \%$ considera que la empresa esta en capacidad de competir con otras industrias (Gráfica 3). 


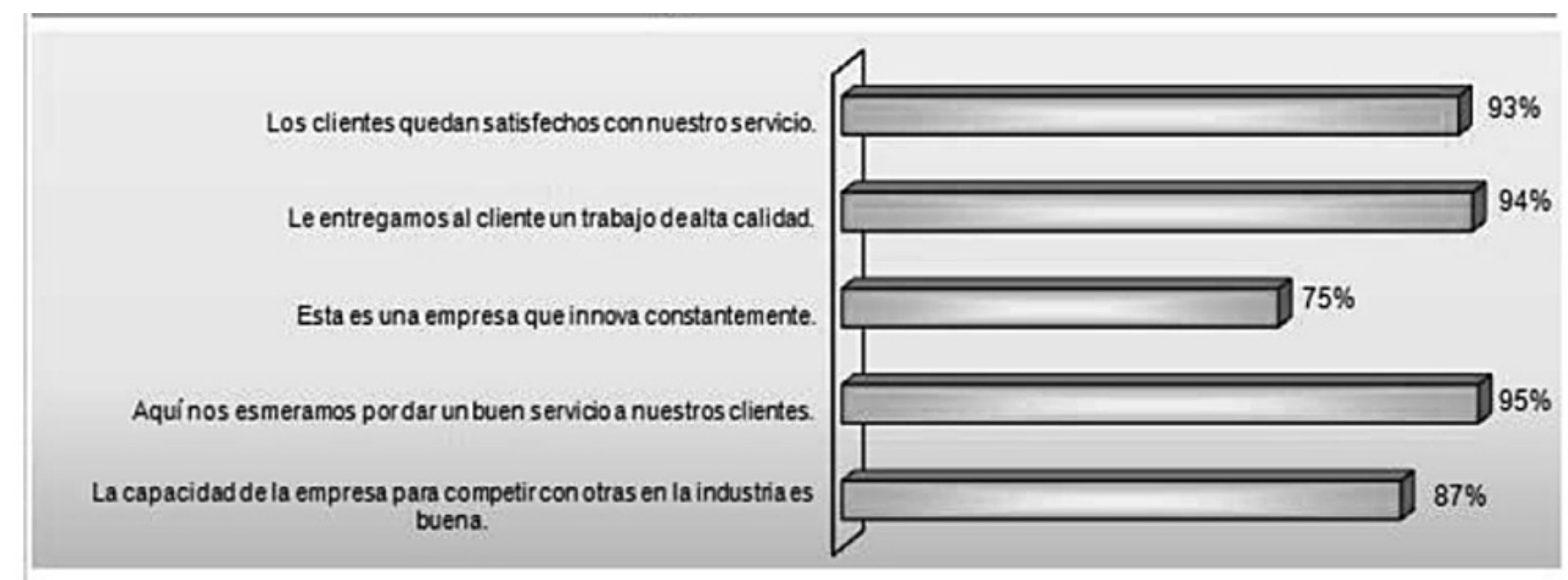

\section{Gráfica 3. Resultados sobre Clientes Externos}

\section{Condiciones de trabajo}

En esta variable se tienen en cuenta los aspectos de salud ocupacional, instrumentación, administración, reconocimiento y capacitación. Dicha variable se desarrolló en las preguntas 20-52. Los aspectos más relevantes son los siguientes: el $85 \%$ de los encuestados considera que la empresa se preocupa por realizar controles

\section{Fuente: Construcción de la autora}

frente a los riesgos en el puesto de trabajo, el $45 \%$ opina que la empresa tiene un sistema claro para evaluar el desempeño de los empleados, para el 55\% la responsabilidad en el puesto de trabajo es equitativa con el salario, y el $44 \%$ de los encuestados cree que los trabajadores destacados tienen un buen reconocimiento (Tabla 2). 
Tabla 2. Resultados sobre Condiciones de Trabajo

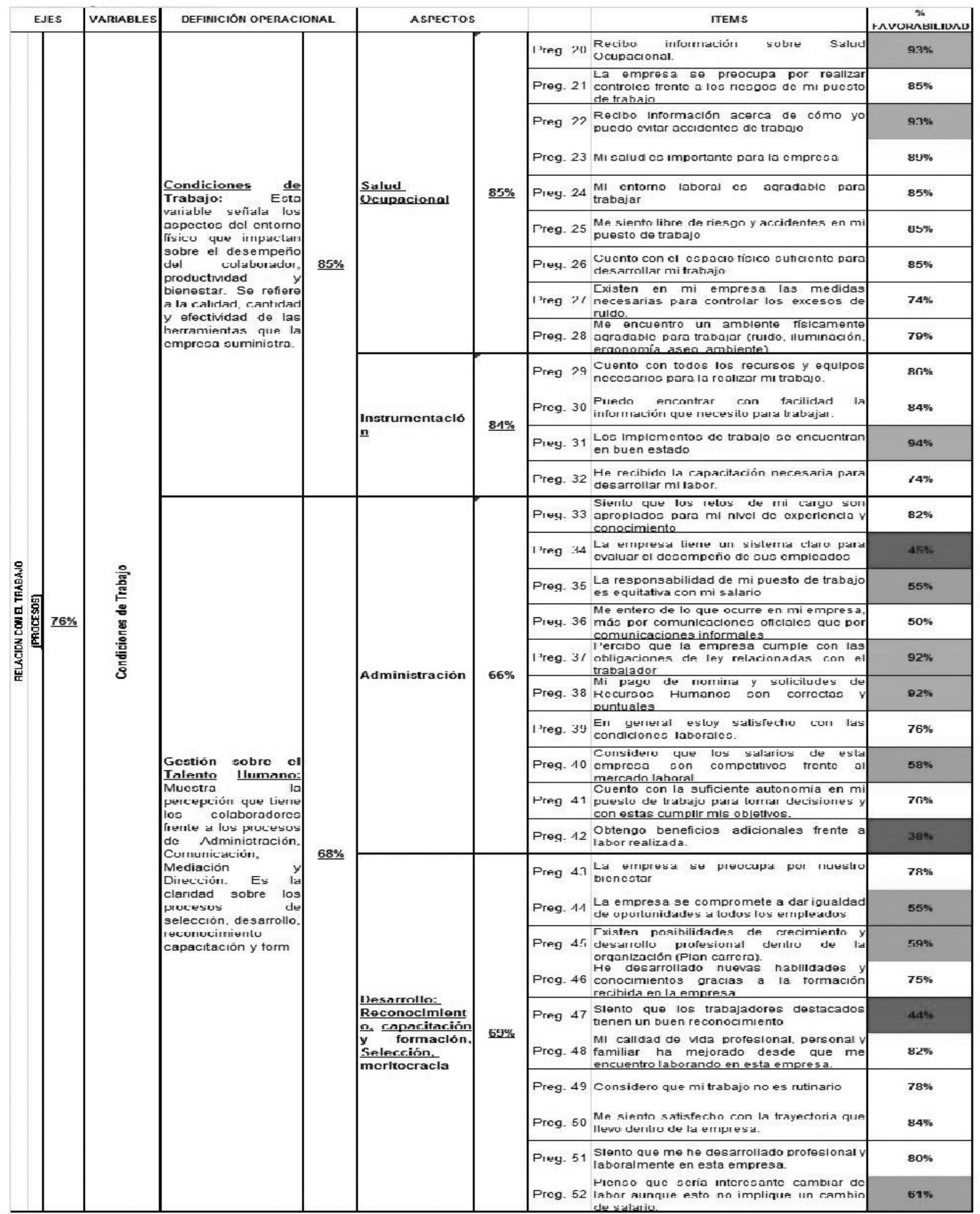

Fuente: Construcción de la autora 
A continuación se presentan la tabla 3 con las respuestas más representativas relacionadas con las condiciones de trabajo.

\section{Tabla 3. Respuestas más representativas relacionadas con las condiciones de trabajo}

\begin{tabular}{|l|l|l|l|l|l|l|l|}
\hline $\begin{array}{l}\text { Pregunta No. 75 ¿Los directivos promueven el } \\
\text { equilibrio entre trabajo y familia como un valor en } \\
\text { la cultura organizacional? }\end{array}$ & \multicolumn{3}{l}{$\begin{array}{l}\text { Pregunta No. 74 iExisten en la empresa programas de } \\
\text { apoyo y asesoramiento frente a problemáticas familiares y } \\
\text { sociales? }\end{array}$} \\
\hline $\begin{array}{l}\text { Opciones de re- } \\
\text { spuesta }\end{array}$ & $\begin{array}{l}\text { No. De } \\
\text { casos }\end{array}$ & $\%$ & $\%$ Acum. & $\begin{array}{l}\text { Opciones de respu- } \\
\text { esta }\end{array}$ & No. De casos & $\%$ & $\%$ Acum. \\
\hline $\begin{array}{l}\text { Totalmente de acu- } \\
\text { erdo }\end{array}$ & 20 & 0,29 & 0,29 & Totalmente de acuerdo & 15 & 0,21 & 0,21 \\
\hline Acuerdo & 6 & 0,09 & 0,37 & Acuerdo & 21 & 0,30 & 0,51 \\
\hline Desacuerdo & 14 & 0,20 & 0,57 & Desacuerdo & 18 & 0,26 & 0,77 \\
\hline $\begin{array}{l}\text { Totalmente en de- } \\
\text { sacuerdo }\end{array}$ & 30 & 0,43 & 1,00 & $\begin{array}{l}\text { Totalmente en desacu- } \\
\text { erdo }\end{array}$ & 16 & 0,23 & 1,00 \\
\hline Total & 70 & 1,00 & 1,00 & Total & 70 & 1,00 & 1,00 \\
\hline
\end{tabular}

Fuente: Construcción de la autora

\section{Relación con las personas}

En esta variable se tienen en cuenta los aspectos de profesionalismo, relaciones de equipo y equilibrio familiatrabajo.

En la parte de profesionalismo, correspondiente a las pre- guntas $67-72$ se evidencia como el $96 \%$ de los encuestados considera que tiene las competencias profesionales para asumir un cargo de mayor responsabilidad, y el $89 \%$ considera que sus ideas facilitan la toma de decisiones dentro de su equipo de trabajo. (Gráfica 4).

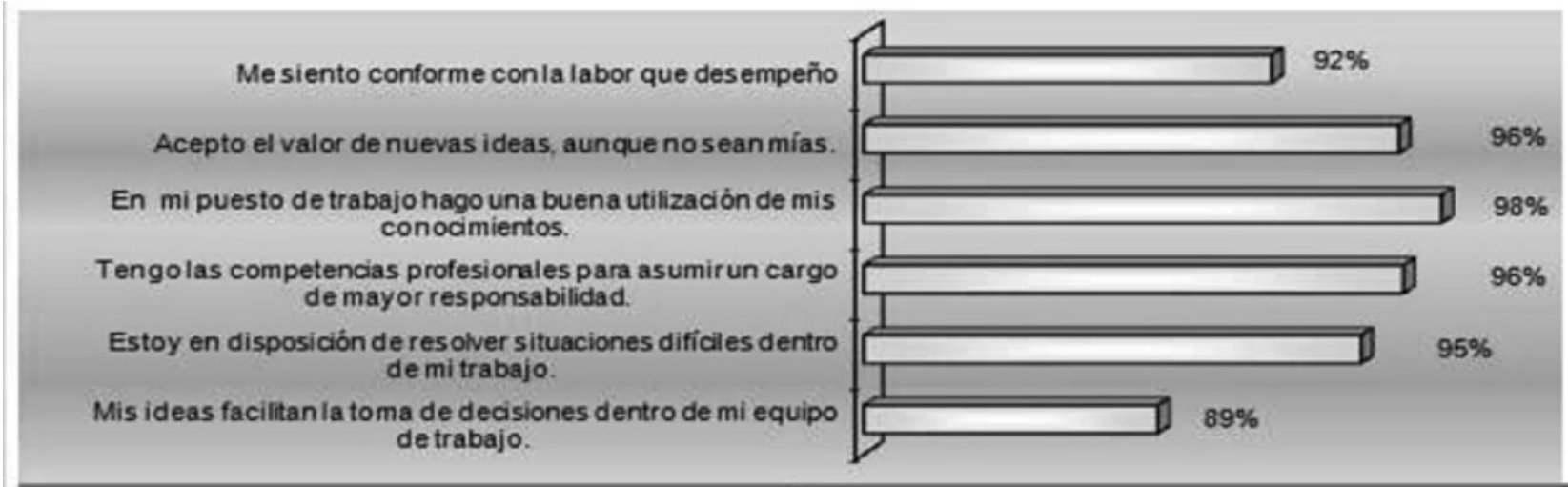

Gráfica 4. Resultados sobre profesionalismo

Fuente: Construcción de la autora

En cuanto a la variable equilibrio familia - trabajo, se encontró, en las respuestas correspondientes a las preguntas $73-77$, que solo el $37 \%$ considera que los directivos promueven el equilibrio entre el trabajo y la familia como un valor de la cultura organizacional, y el $31 \%$ establece como una de sus prioridades favorecer el equilibrio entre familia y trabajo (Gráfica 5). 


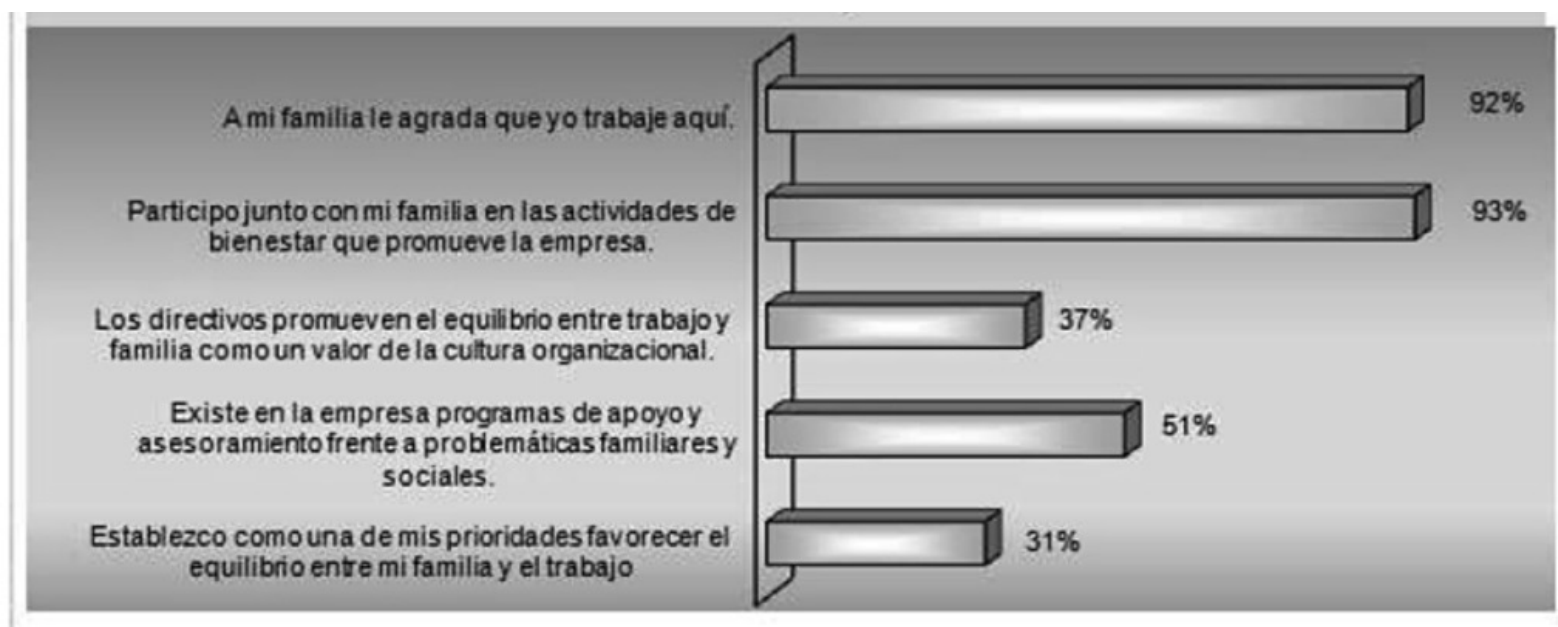

\section{Gráfica 5. Resultados sobre Equilibrio Familia-Trabajo}

\section{Fuente: Construcción de la autora}

A continuación se presenta la tabla 4 con las respuestas màs representativas relacionadas con el equilibrio familia - trabajo

Tabla 4. Respuestas más representativas relacionadas con el equilibrio familia - trabajo

\begin{tabular}{|l|r|l|l|l|l|l|r|}
\hline \multicolumn{4}{|l|}{$\begin{array}{l}\text { Pregunta No. } 22 \text { ¿Recibo información acerca de cómo } \\
\text { puedo evitar accidentes de trabajo? }\end{array}$} & \multicolumn{4}{l|}{$\begin{array}{l}\text { Pregunta No. 34 ¿La empresa tiene un sistema claro } \\
\text { para evaluar el desempeño de sus empleados? }\end{array}$} \\
\hline Opciones de respuesta & $\begin{array}{l}\text { No. De } \\
\text { casos }\end{array}$ & $\%$ & $\%$ Acum. & Opciones de respuesta & $\begin{array}{l}\text { No. De } \\
\text { casos }\end{array}$ & $\%$ & $\%$ Acum. \\
\hline Totalmente de acuerdo & 38 & 0,54 & 0,54 & Totalmente de acuerdo & 9 & 0,13 & 0,13 \\
\hline Acuerdo & 27 & 0,39 & 0,93 & Acuerdo & 22 & 0,31 & 0,44 \\
\hline Desacuerdo & 5 & 0,07 & 1,00 & Desacuerdo & 12 & 0,17 & 0,61 \\
\hline $\begin{array}{l}\text { Totalmente en desacu- } \\
\text { erdo }\end{array}$ & 0 & 0,00 & 1,00 & $\begin{array}{l}\text { Totalmente en desacu- } \\
\text { erdo }\end{array}$ & 27 & 0,39 & 1,00 \\
\hline Total & 70 & 1,00 & 1,00 & Total & 70 & 1,00 & 1,00 \\
\hline
\end{tabular}

Fuente: Construcción de la autora

\section{Discusión}

Según los resultados de la encuesta aplicada se puede interpretar lo siguiente:

En la variable de relación con la empresa se evidencia que hay un bajo grado de conocimiento y de identidad del trabajador con las metas de la organización, pues los encuestados no perciben que la empresa tenga en cuenta los intereses ni las preocupaciones de los empleados para tomar decisiones importantes.

En cuanto a la imagen corporativa, hace evidente que los trabajadores están integrados a la compañía, que muestran su afiliación y su visión de la empresa frente a su competencia directa.

Los colaboradores de la empresa perciben una buena relación generada por la empresa hacia sus clientes, a quienes brinda calidad, satisfacción, atención a sus requerimientos e innovación permanente.

Los resultados en cuanto a la variable de relación con el trabajo señalan que los aspectos del entorno físico impactan positivamente sobre el desempeño del trabajador, su productividad y su bienestar. Los encuestados consideran adecuadas la cantidad, la calidad y la efectividad de las herramientas que la empresa les suministra para desempeñar sus funciones.

En cuanto a la gestión del talento humano, la percepción que tienen los trabajadores no es muy favorable frente a los procesos de administración, comunicación, mediación y dirección. Consideran que los procesos de reconocimiento, igualdad de oportunidades y posibilidades de crecimiento y desarrollo profesional no son claros. 
En la variable de relaciones entre personas se evidencian buenas relaciones entre los diferentes niveles de la organización, atendiendo a la comunicación, la integración, la emocionalidad y el reconocimiento. Además, hay un alto nivel de profesionalismo realizando aportes a la compañía mas allá de las funciones establecidas.

Sobre el aspecto de equilibro familia- trabajo los resultados reflejan una baja capacidad de la organización para fortalecer la relación del colaborador con su núcleo familiar, ya que los directivos no promueven el equilibrio familia- trabajo como un valor de la cultura organizacional.

\section{Conclusiones}

El estudio realizado permitió conocer cómo es percibida la organización por parte de sus empleados, si su filosofía es comprendida y compartida por su personal, qué problemas de comunicación se presentan en ella, cómo funcionan las diferentes relaciones que se establecen entre el personal y sus jefes.

Sin lugar a dudas, los factores más destacados durante el desarrollo de esta investigación fueron la motivación y la integración, estas permiten un mejor desempeño, incrementan la productividad, así como el rendimiento de las labores de una manera más eficiente y efectiva para la organización; y si los empleados perciben bienestar en medio de sus esfuerzos, se destacará la satisfacción laboral.

Los resultados pueden sugerir que existe un adecuado clima laboral en la empresa Energías S. A.; el mayor porcentaje de percepción a ese respecto lo tienen el profesionalismo, los clientes externos, las condiciones de trabajo y las relaciones de equipo.

Existe alta probabilidad de intervención en las áreas donde se encuentran variables por debajo del $75 \%$ de favorabilidad; tales como la orientación organizacional, la gestión del talento humano y el equilibrio familia- trabajo

Estas variables se pueden moldear para satisfacer adecuadamente las necesidades de los colaboradores de la empresa, y así aumentar la productividad, la eficacia y la competitividad. Se pueden crear programas que reconozcan las labores de los empleados y brinden la oportunidad de reconocimientos y de beneficios adicionales para el trabajador. Además, es importante fomentar programas de apoyo y de asesoramiento frente a las problemáticas familiares y sociales.

\section{Referencias bibliográficas}

AA.VV, (2010). Administración, decima edición, México, Prentice-Hall, p 53-54.

Angura, M. \& Arnau, J. (1995). Métodos de investigación en psicología. España: Editorial Síntesis.
Álvarez E. y Ortiz. (2002). Asesorías para el Diagnóstico e Intervención del Clima Organizacional. Trabajo de Grado, Maestría en Ciencias Sociales, Universidad Javeriana. Colombia.

Anderson, L. (2000). El Clima Organizacional y la Satisfacción Laboral. Trabajo de Grado. México.

Bouthillier F. y Shearer K., (2002). Understanding knowledge management and information management: the need for an empirical perspective

Brunet L (1999). El Clima de Trabajo en las Organizaciones: Definiciones, diagnóstico y consecuencias. México: Editorial Trillas.

Castañeda, A. (2001). Las nuevas tecnologías de la información y las comunicaciones como proceso cultural y las bases para su impacto en la actividad educativa. Universidad Técnica de Ambato. Perú.

Daniels Aubrey C, (2000) Gerencia de desempeño, Refuerzos positivos, 3 edición, México, Mc Graw Hill, pag $58-67$

DeStefano, T.J., Clark, H., Gavin, M. y Potter, T. (2005). The relationship between work environment factors and job satisfaction among rural behavioral health professionals. Journal of community psychology, 1-7.

Douglas A. Ready, (2010)“¿Tiene usted un alto potencial?, Edición 42, p. 48-53

Durán, P. (2003). La Importancia del Clima Organizacional en el Éxito de las Empresas. Chile.

García García, I. y Sánchez Santa-Bárbara, E. (2008). Clima psicológico y estilos de liderazgo. Revista de Trabajo y Seguridad Social. CEF, 299: 217-264.

Garzón, M. (2005). El desarrollo Organizacional y el cambio planeado, primera edición, Bogotá, Centro Editorial Universidad del Rosario, $\mathrm{p}$ 81-90

Gongalves, A. (1997). Dimensiones del Clima Organizacional, Sociedad Latinoamericana para la Calidad (SLC).

Jaime Santana, P. y Araujo Cabrera, Y. (2009). Clima y cultura organizacional: ¿Dos constructos para explicar un mismo fenómeno? Decisiones organizativas, 296324.

Jensen, A.J. y A.P. Sage (2000). A Systems Management Approach for Improvement of Organizational Performance Measurement Systems. Information Knowledge Systems Management.

Marín Pérez, M. (2003). Relación entre clima y el compromiso organizacional en una empresa del Sector Petroquímico. Tesis de Licenciatura. Universidad Católica Andrés Bello, Facultad de Ciencias Económicas y Sociales, Caracas, Venezuela. 
Martínez, R. (2002). El Éxito es de Quienes Pueden Cambiar el Clima. HayGroup. Austria.

Mendez, Carlos (2006). Metodología. México: McGraw-Hill.
Robbins, Stephen P. (1999) Comportamiento Organizacional, Ed. Prentice Hall, México.

Villán, B. (2000). Implementación de instrumentos para la gestión de la información. Editorial ABZ, México.

\section{ANEXO 1. ENCUESTA A LOS EMPLEADOS DE ENERGÍAS S. A.}

\section{Energias S.A}

\section{ENCUESTA DE CLIMA ORGANIZACIONAL}

Las preguntas que encuentra a continuación nos darán a conacer las percepciones y sentimientos del personal respecto a su entorno laboral, con el fin de orientar la labor de Gestión Humana en el mejoramienta continuo del bienestar de sus colaboradores.

\section{DATOS INICIALES}

1. Dependencid $y / u$ proyeclo dunde labora:

2. Grupo Poblacional al que pertenece:

O Nivel Directivo

O Supervisor

O Nivel Profesional

O Nivel Técnico

O Nivel Asistenclal

3. Estado Civil:

u soltero

U Casado / Unıón Libre

U separado / viudo

4. Gènero:

O Femenino

u Masculıno

\begin{tabular}{|c|c|}
\hline $\begin{array}{l}\text { 5. Antigüedad en la empresa } \\
\text { a Menos de } 1 \text { año }\end{array}$ & $\begin{array}{l}\text { 8. Area } \\
\text { O Finanzas }\end{array}$ \\
\hline O Entre 1 y 5 años & O Pracura \\
\hline a más de 5 afios & O Recursos Humanos \\
\hline 6. Pals & O HSEQ/SHA \\
\hline o Argentına & $0 \pi$ \\
\hline a Culurnbia & O Administrativo \\
\hline o Espafa & U Uperacions Hıdrocarburos \\
\hline a Panama & U Uperacianes Infraestructura \\
\hline o otros & U Mınerıa \\
\hline $\begin{array}{l}\text { f. Uivision } \\
\text { u Hidrocarburas }\end{array}$ & U Manufactura \\
\hline u is inelectra & \\
\hline $\mathbf{u}$ is Gomez & \\
\hline
\end{tabular}

Calificación: Califique las siguientes afirmaciones de acuerdo a su forma de sentir, pensar o actuar, donde 4 es la calificación más alta y 1 la más baja. Marque una sola posibilidad por afirmación.
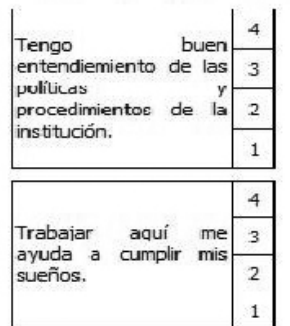

\begin{tabular}{|l|l|}
\hline \multirow{2}{*}{ Aquí nos esmeramos } & 4 \\
\cline { 2 - 2 } & 3 \\
\hline
\end{tabular} \begin{tabular}{ll|l} 
Aquí nos esmeramos & 4 \\
por dar un buen 3 & \\
& &
\end{tabular} servido a nuestros dientes.

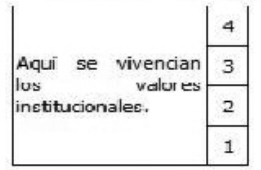

\begin{tabular}{|l|l|}
\hline \multirow{2}{*}{ Ayuí vale la pena } & 4 \\
\cline { 2 - 2 } & 3 \\
\cline { 2 - 2 }
\end{tabular}

dar lo mejor de mí.
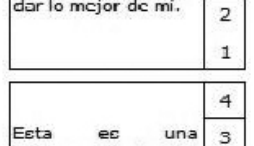

empresa que innova 3

constantemente.

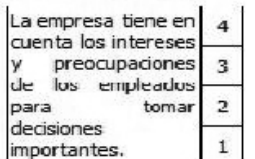

importantes.
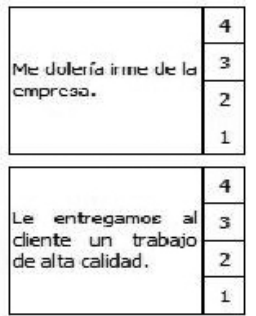

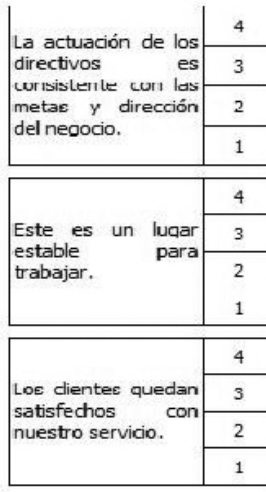

SEGUNDA PARTE

Calificación: Califique seqún la siquiente descripciốn: T.A. = Totalmente de Acuerdo $\quad$ A. = Acuerdo D = Desacuerdo T.D. = Totalmente en Desacuerdo

\begin{tabular}{|l|c|}
\hline \multirow{2}{*}{$\begin{array}{l}\text { Recibo información } \\
\text { sobre } \\
\text { Ocupacional. Salud }\end{array}$} & TA \\
\cline { 2 - 2 } & $\mathrm{D}$ \\
\cline { 2 - 2 } & $\mathrm{T}$ \\
\hline
\end{tabular}

TA Cuento con todos los recursos y equipos A \begin{tabular}{ll|l|}
$\begin{array}{ll}\text { necesarios para la } \\
\text { realizar mi trabajo }\end{array}$ & D \\
\cline { 2 - 3 } & & TD \\
\hline
\end{tabular}

\begin{tabular}{|l|l|}
\hline & $T A$ \\
\cline { 2 - 2 } & \\
\hline
\end{tabular} sistema daro un A evaluar el desempeño de sus empleados

\section{\begin{tabular}{|l|l|}
\hline & TD \\
\hline
\end{tabular}}

La empreca se $T A$ compromete a dar $\wedge$ igualdad oportunidades

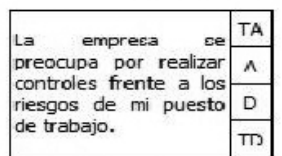

de trabajo. TI

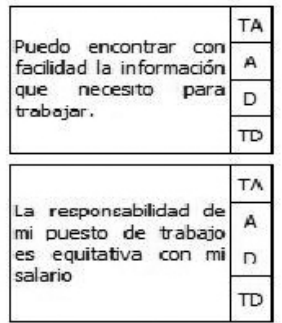

\begin{tabular}{|l|l|}
\hline Existen posibilidades & TA \\
\hline
\end{tabular} \begin{tabular}{ll|l} 
de crecimiento y & $y$ \\
desarrollo profesional & $\wedge$
\end{tabular} dentro de la profional organisac carrera).

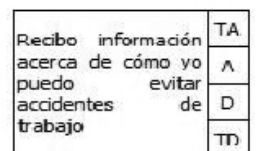

\begin{tabular}{|l|l}
\hline & $T n$ \\
\hline
\end{tabular}

\begin{tabular}{|l|c|}
\hline \begin{tabular}{l|l|} 
Los implementos de \\
trabajo \\
encuentran en buen
\end{tabular} & TA \\
\cline { 2 - 2 } \begin{tabular}{l} 
estado \\
\cline { 2 - 2 }
\end{tabular} & D \\
\cline { 2 - 3 } & TD \\
\hline
\end{tabular}

\begin{tabular}{|l|l|}
\hline Me entero de lo que & TA \\
\hline
\end{tabular} ocurre en mi empresa, más por comunicaciones oficiales que por $n$ \begin{tabular}{l|l|}
$\begin{array}{l}\text { comunicaciones } \\
\text { informales }\end{array}$ & TD \\
\cline { 2 - 3 }
\end{tabular}

\begin{tabular}{|l|l|}
\hline He desarrollado & TA
\end{tabular} nuevas habilidades y conocimienos A \begin{tabular}{lll|l} 
gracias & $a$ & la \\
formatión & recihida & D
\end{tabular} $\begin{array}{ll}\text { formación recihida } & \text { D } \\ \text { en la empresa } & \text { Tn }\end{array}$
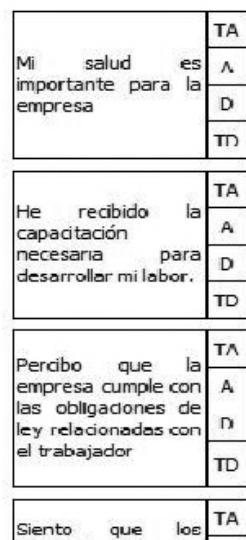

\begin{tabular}{ll|l}
$\begin{array}{l}\text { Siento que los } \\
\text { trabajadores }\end{array}$ & $\wedge$
\end{tabular}

destacados tienen

destacadas tienen
un buen
reconocamiento

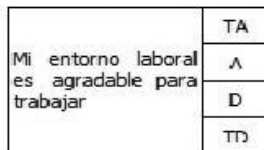

\begin{tabular}{|l|l|}
\hline Siento que los retos & $\mathrm{S}$ \\
\hline
\end{tabular} dc mi cargo son CS \begin{tabular}{l|l} 
anronianns nara mi & \\
nivel de experencia & $\mathrm{CN}$
\end{tabular} y canocimiento

\begin{tabular}{|l|c|}
\hline Y canocimiento & N \\
\hline \multirow{2}{*}{$\begin{array}{l}\text { La empresa se por } \\
\text { preocupa } \\
\text { nuestro bienestar }\end{array}$} & $\mathrm{A}$ \\
\cline { 2 - 2 } & $\mathbf{n}$ \\
\cline { 2 - 2 } & $\mathrm{TD}$ \\
\hline \multirow{2}{*}{$\begin{array}{l}\text { Me siento libre de } \\
\text { rlesgo de acadentes } \\
\text { cn mi pucsto dc }\end{array}$} & $\mathrm{TA}$ \\
\cline { 2 - 2 } trahajn & $\mathbf{D}$ \\
\hline
\end{tabular}




\begin{tabular}{|c|c|c|c|c|c|c|c|c|c|}
\hline Calificació & & $\begin{array}{c}\text { fique según la siguier } \\
\text { C.N. }\end{array}$ & ite & $\begin{array}{l}\text { ripción: } \\
\text { lunca }\end{array}$ & $e_{N}$ & Nunco & c.s & $=$ Casi Siempre & \\
\hline \multirow{3}{*}{ 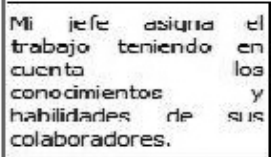 } & \multirow{3}{*}{\begin{tabular}{|c|}
$\mathrm{S}$ \\
$\mathrm{CS}$ \\
$\mathrm{CN}$ \\
$\mathrm{N}$ \\
\end{tabular}} & \multirow{3}{*}{$\begin{array}{l}\text { Mi jefe motiva a su } \\
\text { cquipo para que } \\
\text { mejore sue habilidades } \\
\text { y ranncimientos. }\end{array}$} & $\mathrm{s}$ & \multirow{3}{*}{$\begin{array}{lr}\text { Mi jefe tiene los } \\
\text { conocimicntos r y } \\
\text { destrezas } & \text { para } \\
\text { dirigir mi Area. } & \end{array}$} & $s$ & \multirow{3}{*}{$\begin{array}{l}\text { Con mi jefc pucdo } \\
\text { hablar } \\
\text { abiertamente. }\end{array}$} & $s$ & \multirow{3}{*}{$\begin{array}{l}\text { Mi jcfic valora los } \\
\text { aportes para lograr } \\
\text { mejoras en el área. }\end{array}$} & $s$ \\
\hline & & & cs & & es & & Cs & & CS \\
\hline & & & $\begin{array}{c}\mathrm{CN} \\
\mathrm{N}\end{array}$ & & $\begin{array}{c}C N \\
N\end{array}$ & & $\begin{array}{l}\mathrm{N} \\
\mathrm{N}\end{array}$ & & $\begin{array}{l}\mathrm{CN} \\
\mathrm{N}\end{array}$ \\
\hline \multirow{4}{*}{$\begin{array}{l}\text { Si me fuera a otra } \\
\text { empresa, extrañaria a } \\
\text { mis comparieros. }\end{array}$} & $\varepsilon$ & \multirow{4}{*}{$\begin{array}{l}\text { La personas nuevas en } \\
\text { la empresa emcientran } \\
\text { un ambiente de fäcl } \\
\text { integración. }\end{array}$} & $s$ & \multirow{4}{*}{ 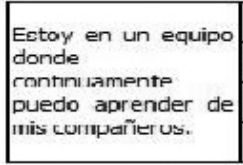 } & $s$ & \multirow{4}{*}{$\begin{array}{ll}\text { Valaramos los los } \\
\text { distintos puntos de } \\
\text { vistar } \\
\text { conocamientos de los } \\
\text { integrantes r del } \\
\text { equipo. }\end{array}$} & $s$ & \multirow{4}{*}{$\begin{array}{l}\text { Cuando nos delegan } \\
\text { tiareas, mmn equinn } \\
\text { las cumplimos } \\
\text { efectivamente. }\end{array}$} & $s$ \\
\hline & $\mathrm{Cs}$ & & Cs & & $\mathrm{Cs}$ & & CS & & CS \\
\hline & $\mathrm{CN}$ & & $\mathrm{CN}$ & & $\mathrm{CN}$ & & $\mathrm{CN}$ & & $\mathrm{CN}$ \\
\hline & $\mathrm{N}$ & & $N$ & & $N$ & & $\mathrm{~N}$ & & $N$ \\
\hline \multirow{4}{*}{$\begin{array}{l}\text { Mis ideas facilitan la } \\
\text { toma de decisiones } \\
\text { dentro de mi equipo de } \\
\text { trabajo. }\end{array}$} & $s$ & \multirow{4}{*}{$\begin{array}{l}\text { Estoy en disposiciōn de } \\
\text { resolver situadanes } \\
\text { dificiles dentra de mi } \\
\text { trabujo. }\end{array}$} & $s$ & \multirow{4}{*}{$\begin{array}{l}\text { Tengn } \\
\text { competencaas las } \\
\text { profesionales para } \\
\text { asumir un cargo de } \\
\text { mayor } \\
\text { responsabilidad. }\end{array}$} & $s$ & \multirow{4}{*}{$\begin{array}{l}\text { En mı puesto de } \\
\text { trabajo hago una } \\
\text { buena utilización de } \\
\text { mis conacimicntos. }\end{array}$} & $s$ & \multirow{4}{*}{$\begin{array}{l}\text { Acepto el valor de } \\
\text { nuevas } \\
\text { aunque no seas, } \\
\text { mins. }\end{array}$} & $s$ \\
\hline & cs & & cs & & cs & & cs & & $\operatorname{cs}$ \\
\hline & $\mathrm{CN}$ & & $\mathrm{CN}$ & & $\mathrm{CN}$ & & $\mathrm{CN}$ & & CN \\
\hline & $N$ & & $\mathrm{~N}$ & & $N$ & & $\mathrm{~N}$ & & $\mathrm{~N}$ \\
\hline \multirow{4}{*}{$\begin{array}{l}\text { Establezco como una } \\
\text { de mis prioridades } \\
\text { favorccer cl cquilibrio } \\
\text { entre mi familia y el } \\
\text { trabajo }\end{array}$} & s & \multirow{4}{*}{$\begin{array}{l}\text { Existe en la empresa } \\
\text { programas de apoyro y } \\
\text { oscsoramicnto fronte a } \\
\text { problemáticas } \\
\text { tamiliares y saciales. }\end{array}$} & $s$ & \multirow{4}{*}{$\begin{array}{l}\text { Los directivos } \\
\text { promueven el } \\
\text { equilibria entre } \\
\text { trabajo y familia } \\
\text { como un valor de la } \\
\text { cultura } \\
\text { organizanonal. }\end{array}$} & $s$ & \multirow{4}{*}{ 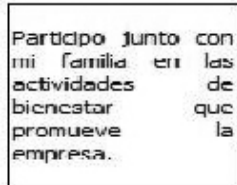 } & $s$ & \multirow{4}{*}{$\begin{array}{l}\text { A mi familia le } \\
\text { agrada que yo } \\
\text { trabaje aquí. }\end{array}$} & $s$ \\
\hline & cs & & $\operatorname{cs}$ & & cs & & CS & & cs \\
\hline & $\mathrm{CN}$ & & $\mathrm{CN}$ & & $C N$ & & $\mathrm{CN}$ & & $\mathrm{CN}$ \\
\hline & $\mathrm{N}$ & & $\mathrm{N}$ & & N & & $\mathrm{N}$ & & $\mathrm{N}$ \\
\hline \multirow{4}{*}{$\begin{array}{l}\text { Recomendaria } \\
\text { empresa como lugar de } \\
\text { trahajo a mis amigns }\end{array}$} & 5 & & 5 & & 5 & & 5 & & 5 \\
\hline & $\operatorname{cs}$ & 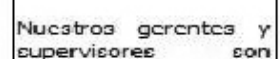 & $c s$ & $\left|\begin{array}{ll}\text { Mi paga de nomina y } \\
\text { solicitudes de de }\end{array}\right|$ & $\operatorname{cs}$ & En gencral cstoy & $c s$ & Mi calidad de vida & $c s$ \\
\hline & $\mathrm{CN}$ & $\begin{array}{l}\text { huienos de estandires } \\
\text { de servidio al cluente. }\end{array}$ & $\mathrm{CN}$ & $\left|\begin{array}{lll}\text { Recoursos } & \text { Humanos } \\
\text { san rorrertas } & \text { yn }\end{array}\right|$ & $\mathrm{CN}$ & \begin{tabular}{l|} 
Ennrlicinnes \\
laborales
\end{tabular} & $C N$ & $\begin{array}{l}\text { tamiliar } \\
\text { mejorando estan }\end{array}$ & $\mathrm{CN}$ \\
\hline & $\mathrm{N}$ & & $\mathrm{N}$ & & $\mathrm{N}$ & & $\mathrm{N}$ & & N \\
\hline & 3 & & 3 & & $\mathrm{~s}$ & & s & & $\mathrm{s}$ \\
\hline $\begin{array}{l}\mathrm{Dc} \text { habcr sabido como } \\
\text { iban a ser las coesas en }\end{array}$ & cs & ESe siente integrado & $\operatorname{cs}$ & $\begin{array}{l}\text { Conoce bicn quc } \\
\text { aporta eu trabajo al }\end{array}$ & $\mathrm{cs}$ & 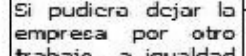 & $\mathrm{cs}$ & Tiene expacio & $\operatorname{cs}$ \\
\hline $\begin{array}{l}\text { su empresa, hubiera } \\
\text { ingresado en ella? }\end{array}$ & $\mathrm{CN}$ & en la empresa? & $\mathrm{CN}$ & $\begin{array}{l}\text { conjurta de la } \\
\text { empresa? }\end{array}$ de & CN & $\begin{array}{l}\text { trabajo, a igualdad } \\
\text { de remuneraciones, }\end{array}$ & $\mathrm{CN}$ & $\begin{array}{l}\text { suticiente en su } \\
\text { puesto de trabajo? }\end{array}$ & CN \\
\hline & $\mathrm{N}$ & & $\mathrm{N}$ & & $\mathrm{N}$ & & $\mathrm{N}$ & & $\mathrm{N}$ \\
\hline & 5 & Su impresora está & $\mathrm{s}$ & Depende por & $s$ & & $s$ & & $s$ \\
\hline Existe un nivel de ruldo & es & $\begin{array}{l}\text { demasiado lejos de vd } \\
\text { y le obliqa a levantarse }\end{array}$ & es & habitualmente, para & es & $\mid \begin{array}{cr}\text { Prefiere } & \text { cumplir } \\
\text { ordenes } & \text { slempre }\end{array}$ & es & siente como una & es \\
\hline $\begin{array}{l}\text { Imulesilu En su vuesil } \\
\text { de trabajo? }\end{array}$ & $\mathrm{CN}$ & $\mid \begin{array}{c}\text { Curilinuantinerile, para it } \\
0 \text { recager lo que }\end{array}$ & $\mathrm{CN}$ & lo que le dicen que & $\mathrm{CN}$ & $\begin{array}{l}\text { Lomat in inialivas y } \\
\text { responsabilidades? }\end{array}$ & $\mathrm{CN}$ & $\begin{array}{l}\text { "rubul" } \\
\text { trabojo? }\end{array}$ EIl su & $\mathrm{CN}$ \\
\hline & N & imprime? & $\mathrm{N}$ & cu jefe a jefes? & N & & $\mathrm{N}$ & & $\mathrm{N}$ \\
\hline & $\mathrm{s}$ & & $\mathrm{s}$ & & $\mathrm{s}$ & & $\mathrm{s}$ & & $\mathrm{s}$ \\
\hline Le atrae más el trabajo & cs & Considera que tiene un & $c s$ & Se $\quad$ considera & $\mid c s$ & Cree vd que no & $c s$ & remuneración no lo & $c s$ \\
\hline $\begin{array}{l}\text { que haren ing } \\
\text { compañeros que le }\end{array}$ & \begin{tabular}{|l|}
3 \\
\end{tabular} & $\begin{array}{l}\text { entornn } \\
\text { entre sus companigns }\end{array}$ & $\mathrm{cs}$ & $\begin{array}{l}\text { vigilarn } \\
\text { contrnuamente por }\end{array}$ & & en otra empresa sus & & existen otros & \\
\hline 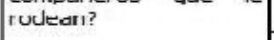 & $\mathrm{CN}$ & Je Lrabaio? & $\mathbf{c N}$ & su iefe $u$ iefes? & CN & $\begin{array}{l}\text { Condidclones } \\
\text { sialatiales ac lualtes? }\end{array}$ & $\mathrm{CN}$ & $\begin{array}{ll}\text { factores } & \text { en } \\
\text { emvitsal yue }\end{array}$ & $\mathrm{CN}$ \\
\hline & $\mathrm{N}$ & & $\mathrm{N}$ & & $\mathrm{N}$ & & $\mathrm{N}$ & componsan? & $\mathrm{N}$ \\
\hline & 3 & & $\mathrm{~s}$ & & 3 & & 3 & & $\mathrm{~s}$ \\
\hline $\begin{array}{l}\text { Cree que existe buena } \\
\text { comunicación de arriba }\end{array}$ & $c s$ & Está satisfecho con su & $\operatorname{cs}$ & & cs & Hace normalmente 0 & $c s$ & & $\operatorname{cs}$ \\
\hline $\begin{array}{l}\text { a abajo, en su } \\
\text { emrresa, entre jetes } y\end{array}$ & $\mathrm{CN}$ & $\begin{array}{l}\text { trayectaria en la| } \\
\text { mmpresa? }\end{array}$ & $\mathrm{CN}$ & en su trabajo? & $\mathrm{CN}$ & $\begin{array}{l}\text { lon } \\
\text { calnor? }\end{array}$ & $C N$ & con trecuencia trio? & CN \\
\hline & $\mathrm{N}$ & & $\mathrm{N}$ & & $\mathrm{N}$ & & $\mathrm{N}$ & & $\mathrm{N}$ \\
\hline & 5 & & $\mathrm{~s}$ & & 5 & & 5 & Piensa que existe & 5 \\
\hline Los servicios $\gamma$ aseos & $\mathrm{Cs}$ & $\left|\begin{array}{lll}5 \mathrm{Lu} & \text { ardenador } \\
\text { computador es muy }\end{array}\right|$ & cs & $\begin{array}{l}\text { Curlsider a VU yue } \\
\text { tiene }\end{array}$ & $\mathrm{cs}$ & $\begin{array}{l}\text { Prefiere cumplit } \\
\text { órdenes siempre a }\end{array}$ & Cs & "lucha" o "puqna" & $\operatorname{cs}$ \\
\hline cstón limpios? & $\mathrm{CN}$ & Vd. continuamente por & $\mathrm{CN}$ & $\begin{array}{l}\text { autonomía cn } \\
\text { trabajo? }\end{array}$ & $\mathrm{CN}$ & $\mid \begin{array}{l}\text { tomar iniciativas } \\
\text { responsabilidades? }\end{array}$ & $\mathrm{CN}$ & $\mid$ & $\mathrm{CN}$ \\
\hline & N & & $\mathrm{N}$ & & N & & $\mathrm{N}$ & mompañerismo & $\mathrm{N}$ \\
\hline & $s$ & & $s$ & & $s$ & & $s$ & & $s$ \\
\hline Uesearia cambiar de & es & $\begin{array}{l}\text { 1 e gustaria traslardarse } \\
a \text { otro centro de }\end{array}$ & es & $\mid \begin{array}{l}\text { ije aru ierdin ron ins } \\
\text { sueldos que existen }\end{array}$ & cs & su remuneración por & $\mathrm{cs}$ & $\begin{array}{l}\text { Sie } \\
\text { buscando onciuentra }\end{array}$ & Cs \\
\hline sirt cambivin la & $\mathrm{CN}$ & $\begin{array}{l}\text { W abaju de su enuresiar } \\
\text { en su misma ciudad a }\end{array} \mid$ & $\mathrm{CN}$ & $\begin{array}{l}\text { Er } 1 \text { su embr esa, u } \\
\text { que deberia de }\end{array}$ & $\mathrm{CN}$ & Eri su Eritorio & $\mathrm{CN}$ & $\begin{array}{l}\text { labur ales wor fuera } \\
\text { de la empresa en }\end{array}$ & $\mathrm{CN}$ \\
\hline rcmuncradion? & $N$ & & $\mathrm{~N}$ & & $N$ & $\begin{array}{l}\text { sompresa? } \\
\text { emplat ac la }\end{array}$ & $\mathrm{N}$ & & $N$ \\
\hline
\end{tabular}

